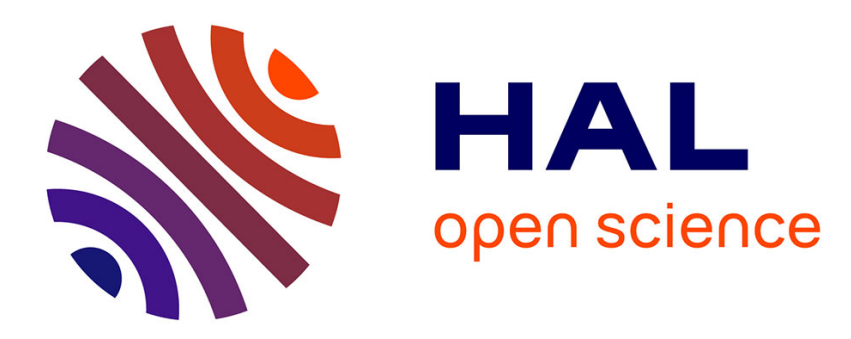

\title{
On a nonlinear system of PDE's arising in free convection
}

\author{
Bernard Brighi, Senoussi Guesmia
}

\section{To cite this version:}

Bernard Brighi, Senoussi Guesmia. On a nonlinear system of PDE's arising in free convection. 2008. hal-00224397

\section{HAL Id: hal-00224397 \\ https://hal.science/hal-00224397}

Preprint submitted on 30 Jan 2008

HAL is a multi-disciplinary open access archive for the deposit and dissemination of scientific research documents, whether they are published or not. The documents may come from teaching and research institutions in France or abroad, or from public or private research centers.
L'archive ouverte pluridisciplinaire HAL, est destinée au dépôt et à la diffusion de documents scientifiques de niveau recherche, publiés ou non, émanant des établissements d'enseignement et de recherche français ou étrangers, des laboratoires publics ou privés. 


\title{
ON A NONLINEAR SYSTEM OF PDE'S ARISING IN FREE CONVECTION
}

\author{
Bernard BRIGHI - Senoussi GUESMIA
}

\section{Introduction}

In this paper, we consider a model problem introduced in [2], and derived from a coupled system of partial differential equation arising in the study of free convection about a vertical flat plate embedded in a porous medium. In [2], some existence result has been obtained under very constraining hypothesis. Here, we come back to the weak formulation of this problem, and prove that there is one and only one weak solution, under reasonable hypotheses on the data.

Details about the physical background can be found, for example, in [13], [14], [15], [16], [19] and [22]. In these papers, the authors assume that convection takes place in a thin layer around the plate. This allows to make boundary-layer approximations, and to get similarity solutions by solving the ordinary differential equation

$$
f^{\prime \prime \prime}+\frac{\mathrm{m}+1}{2} f f^{\prime \prime}-\mathrm{m} f^{\prime 2}=0
$$

on the half line $[0,+\infty)$, with the boundary conditions $f(0)=a, f^{\prime}(0)=1\left(\right.$ or $\left.f^{\prime \prime}(0)=-1\right)$ and $f^{\prime}(t) \rightarrow 0$ as $t \rightarrow+\infty$. These boundary value problems have been widely studied, and mathematical results about them can be found, for example, in [1], [3], [4], [5], [6], [7], [8], [9], [10], [11], [12], [17], [18], [20] and [21].

Let us state now the problem we are interesting in. Let $\Omega$ be a bounded domain of $\mathbb{R}^{2}$ with sufficiently smooth boundary $\Gamma$. Let $\Gamma_{1}$ and $\Gamma_{2}$ be two parts of $\Gamma$, such that mes $\left(\Gamma_{1}\right) \neq 0$ and

$$
\bar{\Gamma}_{1} \cup \bar{\Gamma}_{2}=\Gamma, \Gamma_{1} \cap \Gamma_{2}=\emptyset .
$$

In $\Omega$, we consider the boundary value system defined by

$$
\begin{aligned}
-\Delta \Psi+K . \nabla H & =F \\
-\lambda \Delta H+\nabla H \cdot(\nabla \Psi)^{\perp}+\nabla \Theta \cdot(\nabla \Psi)^{\perp} & =0
\end{aligned}
$$


with mixed boundary conditions for $\Psi$

$$
\Psi=0 \text { on } \Gamma_{1} \quad \text { and } \quad \frac{\partial \Psi}{\partial \nu}=0 \text { on } \Gamma_{2},
$$

and for $H$

$$
H=0 \text { on } \Gamma,
$$

where $\vec{\nu}$ is the unit outward normal vector on $\Gamma$ and $(\nabla \Psi)^{\perp}=\left(\partial_{y} \Psi,-\partial_{x} \Psi\right)$. The unknown functions are $\Psi$ and $H$. The functions $F, \Theta$ and $K$ are given, and we suppose that

$$
F \in L^{2}(\Omega)
$$

and that the function $\Theta$ belongs to $H^{2}(\Omega)$ and satisfies

$$
\Delta \Theta=0 \text { in } \Omega \text {. }
$$

Let us notice that $H^{2}(\Omega) \subset C^{0}(\bar{\Omega})$. For the coefficients $K=\left(k_{1}, k_{2}\right)$, it is assumed that

$$
K \in L^{\infty}(\Omega) \times L^{\infty}(\Omega) .
$$

See [2] for details on the derivation of the problem (1)-(4). In the following, we will denote by $(\cdot, \cdot)$ the $L^{2}(\Omega)$-scalar product, and by $\|\cdot\|$ (resp. $|\cdot|_{2},|\cdot|_{\infty}$ and $|\cdot|_{\infty, \Gamma}$ ) the norm of $H^{1}(\Omega)$ (resp. $L^{2}(\Omega), L^{\infty}(\Omega)$ and $L^{\infty}(\Gamma)$ ). We also denote by $H_{0}^{1}\left(\Omega, \Gamma_{1}\right)$ the subset of functions of $H^{1}(\Omega)$ that vanish on $\Gamma_{1}$.

\section{Weak formulation}

In order to define a variational formulation of the previous problem, let us assume that $\Psi$ and $H$ are classical solutions of (1) and (2) in $\Omega$, such that the boundary conditions (3) and (4) hold. Multiplying (1) and (2) by test functions $u \in H_{0}^{1}\left(\Omega, \Gamma_{1}\right)$ and $v \in H_{0}^{1}(\Omega)$, and integrating on $\Omega$, we get

$$
\int_{\Omega} \nabla \Psi . \nabla u d x+\int_{\Omega} u K . \nabla H d x=\int_{\Omega} F u d x
$$

and

$$
\lambda \int_{\Omega} \nabla H . \nabla v d x+\int_{\Omega} v \nabla H \cdot(\nabla \Psi)^{\perp} d x+\int_{\Omega} v \nabla \Theta .(\nabla \Psi)^{\perp} d x=0 .
$$

If now, we only assume that $\Psi \in H_{0}^{1}\left(\Omega, \Gamma_{1}\right)$ and $H \in H_{0}^{1}(\Omega)$, the third integral in the latter equality is still well defined (this is due to the fact that $\Theta \in H^{2}(\Omega)$ ), whereas, a priori, it is not anymore the case for the second one. 
Let us clarify this point. To this end, for $u, v, w \in H^{1}(\Omega)$ such that $u \nabla v \cdot(\nabla w)^{\perp} \in$ $L^{1}(\Omega)$, let us set

$$
a(u, v, w)=\int_{\Omega} u \nabla v \cdot(\nabla w)^{\perp} d x=\left(u \nabla v,(\nabla w)^{\perp}\right)
$$

and let us show the following results.

Lemma 1. - Let $u, v \in H^{1}(\Omega) \cap L^{\infty}(\Omega)$ such that one of them vanishes on the boundary of $\Omega$. For $w \in H^{1}(\Omega)$ we have

$$
a(u, v, w)=-a(v, u, w) .
$$

In particular, for every $u \in H_{0}^{1}(\Omega) \cap L^{\infty}(\Omega)$ and every $w \in H^{1}(\Omega)$ we have $: a(u, u, w)=0$.

Proof. - For $u, v \in H^{1}(\Omega) \cap L^{\infty}(\Omega)$ and $w \in H^{1}(\Omega)$ the quantities $a(u, v, w)$ and $a(v, u, w)$ are well defined. Since, moreover, $u v \in H_{0}^{1}(\Omega)$, we have :

$$
\begin{aligned}
a(u, v, w)+a(v, u, w) & =\left(u \nabla v+v \nabla u,(\nabla w)^{\perp}\right)=\left(\nabla(u v),(\nabla w)^{\perp}\right) \\
& =-\left(\operatorname{div}\left((\nabla w)^{\perp}\right), u v\right)_{H^{-1}(\Omega), H_{0}^{1}(\Omega)}=0
\end{aligned}
$$

because $\operatorname{div}\left((\nabla w)^{\perp}\right)=0$.

Lemma 2. - Let $u, v \in H_{0}^{1}(\Omega)$. For $w \in H^{2}(\Omega)$ we have

$$
a(u, v, w)=-a(v, u, w) .
$$

In particular, for every $u \in H_{0}^{1}(\Omega)$ and every $w \in H^{2}(\Omega)$ we have : $a(u, u, w)=0$.

Proof. - First, because $H^{1}(\Omega) \hookrightarrow L^{4}(\Omega)$, the quantities $a(u, v, w)$ and $a(v, u, w)$ are well defined for all $u, v \in H_{0}^{1}(\Omega)$ and $w \in H^{2}(\Omega)$. On the other hand, by Lemma 1 , for all $\varphi, \psi \in \mathcal{D}(\Omega)$ and all $w \in H^{2}(\Omega)$, we have : $a(\varphi, \psi, w)=-a(\psi, \varphi, w)$; the conclusion then follows from the density of $\mathcal{D}(\Omega)$ in $H^{1}(\Omega)$.

Taking into account Lemma 1, we can replace the second integral in (7) by

$$
-\int_{\Omega} H \nabla v \cdot(\nabla \Psi)^{\perp} d x
$$

which is well defined, if $H \in L^{\infty}(\Omega)$. Having that in mind, we state the following definition.

Definition 3. - We will say that a couple $(\Psi, H)$ is a WEAK SOLUTION of the problem (1)-(4), if $\Psi \in H_{0}^{1}\left(\Omega, \Gamma_{1}\right)$ and $H \in L^{\infty}(\Omega) \cap H_{0}^{1}(\Omega)$, and if the integral identities

$$
\begin{aligned}
(\nabla \Psi, \nabla u)+(K . \nabla H, u) & =(F, u) \\
\lambda(\nabla H, \nabla v)-a(H, v, \Psi)+a(v, \Theta, \Psi) & =0
\end{aligned}
$$

hold for any $u \in H_{0}^{1}\left(\Omega, \Gamma_{1}\right)$ and for any $v \in H_{0}^{1}(\Omega)$. 


\section{Existence and uniqueness of a weak solution}

\subsection{A priory estimates}

We will need the following lemma.

Lemma 4. - Let $\Psi \in H^{2}(\Omega)$. If $H \in H_{0}^{1}(\Omega)$ satisfies

$$
\forall v \in H_{0}^{1}(\Omega), \quad \lambda(\nabla H, \nabla v)-a(H, v, \Psi)=-a(v, \Theta, \Psi),
$$

then

$$
\inf _{\Gamma} \Theta \leq H+\Theta \leq \sup _{\Gamma} \Theta
$$

and

$$
\inf _{\Gamma} \Theta-\sup _{\Omega} \Theta \leq H \leq \sup _{\Gamma} \Theta-\inf _{\Omega} \Theta .
$$

In particular, we have $H \in L^{\infty}(\Omega)$ and $|H|_{\infty}$ is bounded independently of $\Psi$.

Proof. - The ingredients of the proof are in [2, Proposition 3.2] ; for convenience, and because the hypotheses are slightly different, we write it here. Let us set $l=\sup _{\Gamma} \Theta$ and $H^{+}=\sup \{H+\Theta-l ; 0\}$. Since $H^{+} \in H_{0}^{1}(\Omega)$, then (5), (8) and Lemma 2 imply

$$
\begin{aligned}
\lambda\left(\nabla H^{+}, \nabla H^{+}\right) & =\lambda\left(\nabla H, \nabla H^{+}\right)+\lambda\left(\nabla \Theta, \nabla H^{+}\right)=\lambda\left(\nabla H, \nabla H^{+}\right) \\
& =a\left(H, H^{+}, \Psi\right)-a\left(H^{+}, \Theta, \Psi\right) \\
& =a\left(H, H^{+}, \Psi\right)+a\left(\Theta, H^{+}, \Psi\right) \\
& =a\left(H+\Theta, H^{+}, \Psi\right)=a\left(H^{+}, H^{+}, \Psi\right)=0 .
\end{aligned}
$$

It follows that $\left|\nabla H^{+}\right|_{2}=0$ and hence $H^{+}=0$. This gives the second inequality of (9). To obtain the other one, we set $l^{\prime}=\inf _{\Gamma} \Theta$ and $H^{-}=\inf \left\{H+\Theta-l^{\prime} ; 0\right\}$ and proceed in the same way. The inequalities (10) follow immediately from (9).

\subsection{A contraction}

Let $\mathbf{W}=H_{0}^{1}\left(\Omega, \Gamma_{1}\right) \times H_{0}^{1}(\Omega)$. On $\mathbf{W}$ we define the norm \|\|$_{\mathbf{w}}$ by

$$
\|(\Psi, H)\|_{\mathbf{w}}=\kappa|\nabla \Psi|_{2}+|\nabla H|_{2}
$$

where $\kappa>0$ is a constant that we will choose later. 
Let $\mathbf{D}=\mathcal{D}\left(\Omega, \Gamma_{1}\right) \times \mathcal{D}(\Omega)$ and let $\mathbf{F}: \mathbf{D} \rightarrow \mathbf{W}$ be the application defined in the following way. If $(\Psi, H) \in \mathbf{D}$, then $\mathbf{F}(\Psi, H)=(\tilde{\Psi}, \tilde{H})$ where $\tilde{\Psi}$ and $\tilde{H}$ are the unique solutions of the linear problems

$$
\begin{aligned}
\forall u \in H_{0}^{1}\left(\Omega, \Gamma_{1}\right), \quad(\nabla \tilde{\Psi}, \nabla u)=-(K . \nabla H, u)+(F, u), \\
\forall v \in H_{0}^{1}(\Omega), \quad \lambda(\nabla \tilde{H}, \nabla v)-a(\tilde{H}, v, \Psi)=-a(v, \Theta, \Psi) .
\end{aligned}
$$

Let us notice that the coercivity on $H_{0}^{1}(\Omega)$ of the bilinear form

$$
(\tilde{H}, v) \mapsto \lambda(\nabla \tilde{H}, \nabla v)-a(\tilde{H}, v, \Psi)
$$

follows from Lemma 2. If now $\left(\Psi_{1}, H_{1}\right) \in \mathbf{D}$ and $\left(\Psi_{2}, H_{2}\right) \in \mathbf{D}$, then we deduce from (11) and (12) that, for all $u \in H_{0}^{1}\left(\Omega, \Gamma_{1}\right)$ and for all $v \in H_{0}^{1}(\Omega)$, we have

$$
\begin{aligned}
& \left(\nabla\left(\tilde{\Psi}_{1}-\tilde{\Psi}_{2}\right), \nabla u\right)=-\left(K . \nabla\left(H_{1}-H_{2}\right), u\right), \\
& \lambda\left(\nabla\left(\tilde{H}_{1}-\tilde{H}_{2}\right), \nabla v\right)-a\left(\tilde{H}_{1}, v, \Psi_{1}\right)+a\left(\tilde{H}_{2}, v, \Psi_{2}\right)=-a\left(v, \Theta, \Psi_{1}-\Psi_{2}\right) .
\end{aligned}
$$

Let us choose $u=\tilde{\Psi}_{1}-\tilde{\Psi}_{2}$ and $v=\tilde{H}_{1}-\tilde{H}_{2}$. On one hand, using (6), we get

$$
\left|\nabla\left(\tilde{\Psi}_{1}-\tilde{\Psi}_{2}\right)\right|_{2}^{2}=-\left(K . \nabla\left(H_{1}-H_{2}\right), \tilde{\Psi}_{1}-\tilde{\Psi}_{2}\right) \leq|K|_{\infty}\left|\nabla\left(H_{1}-H_{2}\right)\right|_{2}\left|\tilde{\Psi}_{1}-\tilde{\Psi}_{2}\right|_{2},
$$

and hence

$$
\left|\nabla\left(\tilde{\Psi}_{1}-\tilde{\Psi}_{2}\right)\right|_{2} \leq C|K|_{\infty}\left|\nabla\left(H_{1}-H_{2}\right)\right|_{2}
$$

where $C$ is the Poincaré's constant of $\Omega$. On the other hand, using Lemma 1 , we can write

$$
\begin{aligned}
\lambda\left|\nabla\left(\tilde{H}_{1}-\tilde{H}_{2}\right)\right|_{2}^{2} & =a\left(\tilde{H}_{1}, \tilde{H}_{1}-\tilde{H}_{2}, \Psi_{1}\right)-a\left(\tilde{H}_{2}, \tilde{H}_{1}-\tilde{H}_{2}, \Psi_{2}\right)-a\left(\tilde{H}_{1}-\tilde{H}_{2}, \Theta, \Psi_{1}-\Psi_{2}\right) \\
& =a\left(\tilde{H}_{2}, \tilde{H}_{1}, \Psi_{1}\right)-a\left(\tilde{H}_{2}, \tilde{H}_{1}, \Psi_{2}\right)+a\left(\Theta, \tilde{H}_{1}-\tilde{H}_{2}, \Psi_{1}-\Psi_{2}\right) \\
& =a\left(\tilde{H}_{2}, \tilde{H}_{1}, \Psi_{1}-\Psi_{2}\right)+a\left(\Theta, \tilde{H}_{1}-\tilde{H}_{2}, \Psi_{1}-\Psi_{2}\right) \\
& =a\left(\tilde{H}_{2}, \tilde{H}_{1}-\tilde{H}_{2}, \Psi_{1}-\Psi_{2}\right)+a\left(\Theta, \tilde{H}_{1}-\tilde{H}_{2}, \Psi_{1}-\Psi_{2}\right) \\
& =a\left(\tilde{H}_{2}+\Theta, \tilde{H}_{1}-\tilde{H}_{2}, \Psi_{1}-\Psi_{2}\right) \\
& \leq\left|\tilde{H}_{2}+\Theta\right|_{\infty}\left|\nabla\left(\tilde{H}_{1}-\tilde{H}_{2}\right)\right|_{2}\left|\nabla\left(\Psi_{1}-\Psi_{2}\right)\right|_{2}
\end{aligned}
$$

and thanks to Lemma 4 we arrive to

$$
\left|\nabla\left(\tilde{H}_{1}-\tilde{H}_{2}\right)\right|_{2} \leq \frac{1}{\lambda}|\Theta|_{\infty, \Gamma}\left|\nabla\left(\Psi_{1}-\Psi_{2}\right)\right|_{2} .
$$


Now, the estimates (13) and (14) give

$$
\begin{aligned}
\left\|\mathbf{F}\left(\Psi_{1}, H_{1}\right)-\mathbf{F}\left(\Psi_{2}, H_{2}\right)\right\|_{\mathbf{w}} & =\kappa\left|\nabla\left(\tilde{\Psi}_{1}-\tilde{\Psi}_{2}\right)\right|_{2}+\left|\nabla \tilde{H}_{1}-\tilde{H}_{2}\right|_{2} \\
& \leq \kappa C|K|_{\infty}\left|\nabla\left(H_{1}-H_{2}\right)\right|_{2}+\frac{1}{\lambda}|\Theta|_{\infty, \Gamma}\left|\nabla\left(\Psi_{1}-\Psi_{2}\right)\right|_{2} \\
& \leq \max \left\{\kappa C|K|_{\infty} ; \frac{1}{\kappa \lambda}|\Theta|_{\infty, \Gamma}\right\}\left\|\left(\Psi_{1}, H_{1}\right)-\left(\Psi_{2}, H_{2}\right)\right\|_{\mathbf{w}} .
\end{aligned}
$$

In order to have the best constant, we choose $\kappa=\sqrt{\frac{|\Theta|_{\infty, \Gamma}}{\lambda C|K|_{\infty}}}$ and we obtain

$$
\left\|\mathbf{F}\left(\Psi_{1}, H_{1}\right)-\mathbf{F}\left(\Psi_{2}, H_{2}\right)\right\|_{\mathbf{w}} \leq \sqrt{\frac{C|K|_{\infty}|\Theta|_{\infty, \Gamma}}{\lambda}}\left\|\left(\Psi_{1}, H_{1}\right)-\left(\Psi_{2}, H_{2}\right)\right\|_{\mathbf{w}} .
$$

It follows that $\mathbf{F}: \mathbf{D} \rightarrow \mathbf{W}$ is Lipschitz continuous, with the Lipschitz constant

$$
\beta=\sqrt{\frac{C|K|_{\infty}|\Theta|_{\infty, \Gamma}}{\lambda}} .
$$

Since $\mathbf{D}$ is dense in the Banach space $\mathbf{W}$, the map $\mathbf{F}$ can be extended to $\overline{\mathbf{F}}: \mathbf{W} \rightarrow \mathbf{W}$ which is still Lipschitz continuous, with the same Lipschitz constant $\beta$.

If now $\beta<1$, then $\overline{\mathbf{F}}$ is a contraction and hence has a unique fixed point, say $\left(\Psi_{*}, H_{*}\right)$. Let us show that $\left(\Psi_{*}, H_{*}\right)$ is then the unique weak solution of the problem (1)-(4). By density, there exists a sequence $\left(\Psi_{n}, H_{n}\right) \in \mathbf{D}$ such that $\left(\Psi_{n}, H_{n}\right) \rightarrow\left(\Psi_{*}, H_{*}\right)$ in $\mathbf{W}$. In other words, we have

$$
\Psi_{n} \rightarrow \Psi_{*} \quad \text { and } \quad H_{n} \rightarrow H_{*} \quad \text { in } H^{1}(\Omega) \quad \text { as } n \rightarrow+\infty .
$$

If we set $\left(\tilde{\Psi}_{n}, \tilde{H}_{n}\right)=\mathbf{F}\left(\Psi_{n}, H_{n}\right)$, then

$$
\begin{aligned}
\forall u \in H_{0}^{1}\left(\Omega, \Gamma_{1}\right), \quad\left(\nabla \tilde{\Psi}_{n}, \nabla u\right)=-\left(K . \nabla H_{n}, u\right)+(F, u), \\
\forall v \in H_{0}^{1}(\Omega), \quad \lambda\left(\nabla \tilde{H}_{n}, \nabla v\right)-a\left(\tilde{H}_{n}, v, \Psi_{n}\right)=-a\left(v, \Theta, \Psi_{n}\right) .
\end{aligned}
$$

Since $\left(\tilde{\Psi}_{n}, \tilde{H}_{n}\right) \rightarrow \overline{\mathbf{F}}\left(\Psi_{*}, H_{*}\right)=\left(\Psi_{*}, H_{*}\right)$ in $\mathbf{W}$, by taking the limits as $n \rightarrow+\infty$ in (16), we obtain

$$
\forall u \in H_{0}^{1}\left(\Omega, \Gamma_{1}\right), \quad\left(\nabla \Psi_{*}, \nabla u\right)=-\left(K . \nabla H_{*}, u\right)+(F, u) .
$$

As $n \rightarrow+\infty$, we also have $\left(\nabla \tilde{H}_{n}, \nabla v\right) \rightarrow\left(\nabla H_{*}, \nabla v\right)$ and $a\left(v, \Theta, \Psi_{n}\right) \rightarrow a\left(v, \Theta, \Psi_{*}\right)$, for all $v \in H_{0}^{1}(\Omega)$. It follows that $a\left(\tilde{H}_{n}, v, \Psi_{n}\right)$ has a finite limit as $n \rightarrow+\infty$. To compute this limit, let us extract from $\left(\tilde{H}_{n}\right)$ a subsequence $\left(\tilde{H}_{n_{k}}\right)$ such that

$$
\tilde{H}_{n_{k}}(x) \rightarrow H_{*}(x) \quad \text { a.e. in } \Omega \quad \text { as } k \rightarrow+\infty \text {. }
$$

From Lemma 4 , the sequence $\left(\tilde{H}_{n}\right)$ is bounded in $L^{\infty}(\Omega)$ by some constant $c=c(\Theta)$, and thus $H_{*} \in L^{\infty}(\Omega)$ and we have $\left|H_{*}\right|_{\infty} \leq c$. Therefore, on one hand, from (15), we have

$$
\left|a\left(\tilde{H}_{n}, v, \Psi_{n}-\Psi_{*}\right)\right| \leq c\|v\|\left\|\Psi_{n}-\Psi_{*}\right\| \rightarrow 0 \quad \text { as } n \rightarrow+\infty
$$


and, on the other hand, from the Lebesgue theorem, it holds

$$
a\left(\tilde{H}_{n_{k}}, v, \Psi_{*}\right) \rightarrow a\left(\tilde{H}_{*}, v, \Psi_{*}\right) \quad \text { as } k \rightarrow+\infty
$$

It follows that

$$
a\left(\tilde{H}_{n}, v, \Psi_{n}\right) \rightarrow a\left(\tilde{H}_{*}, v, \Psi_{*}\right) \text { as } n \rightarrow+\infty
$$

and (17) gives

$$
\forall v \in H_{0}^{1}(\Omega), \quad \lambda\left(\nabla H_{*}, \nabla v\right)-a\left(H_{*}, v, \Psi_{*}\right)=-a\left(v, \Theta, \Psi_{*}\right) .
$$

From (18), (19) and the fact that $H_{*} \in L^{\infty}(\Omega)$, we obtain that $\left(\Psi_{*}, H_{*}\right)$ is a weak solution of the problem (1)-(4).

The uniqueness follows from the fact that any weak solution of (1)-(4) is a fixed point of $\overline{\mathbf{F}}$. In fact, let $(\Psi, H)$ be a weak solution of the problem (1)-(4), and $\left(\Psi_{n}, H_{n}\right) \in \mathbf{D}$ be a sequence such that

$$
\Psi_{n} \rightarrow \Psi \quad \text { and } \quad H_{n} \rightarrow H \quad \text { in } H^{1}(\Omega) \quad \text { as } n \rightarrow+\infty \text {. }
$$

Let us set $(\tilde{\Psi}, \tilde{H})=\overline{\mathbf{F}}(\Psi, H)$ and $\left(\tilde{\Psi}_{n}, \tilde{H}_{n}\right)=\mathbf{F}\left(\Psi_{n}, H_{n}\right)$. Arguing as above, we can take the limits as $n \rightarrow+\infty$ in (16)-(17), and we obtain

$$
\begin{aligned}
\forall u \in H_{0}^{1}\left(\Omega, \Gamma_{1}\right), \quad(\nabla \tilde{\Psi}, \nabla u)=-(K . \nabla H, u)+(F, u), \\
\forall v \in H_{0}^{1}(\Omega), \quad \lambda(\nabla \tilde{H}, \nabla v)-a(\tilde{H}, v, \Psi)=-a(v, \Theta, \Psi) .
\end{aligned}
$$

This immediatly gives that $\tilde{\Psi}=\Psi$ and $\tilde{H}=H$.

To summarize, we have proved the following result.

Theorem 5. - Let $C$ be the Poincaré's constant of $\Omega$. If we have

$$
C|K|_{\infty}|\Theta|_{\infty, \Gamma}<\lambda
$$

then problem (1)-(4) has one and only one weak solution.

Acknowledgement The authors would like to thank Professor Michel Chipot for his comments and helpful suggestions.

\section{References}

[1] M. Aiboudi and B. Brighi, On the solutions of a boundary value problem arising in free convection with prescribed heat flux, preprint (http://arxiv.org/abs/0707.1628 ; http://hal.archives-ouvertes.fr/hal-00161672). 
[2] S. Akesbi, B. Brighi and J.-D. Hoernel : Steady free convection in a bounded and saturated porous medium. Proceedings of the 2004 Swiss-Japanese Seminar "Recent advances on Elliptic and Parabolic Issues" World Scientific Publishing Co. Pte. Ltd. (2006) 1-17 (http://arxiv.org/abs/math/0601219).

[3] Z. Belhachmi, B. Brighi and K. Taous, On the concave solutions of the Blasius equation, Acta Math. Univ. Comenian. 69 (2000), no. 2, 199-214.

[4] Z. Belhachmi, B. Brighi and K. Taous, Solutions similaires pour un problème de couche limite en milieux poreux, C. R. Mécanique 328 (2000), 407-410.

[5] Z. Belhachmi, B. Brighi and K. Taous, On a family of differential equations for boundary layer approximations in porous media, European J. Appl. Math. 12 (2001), no. $4,513-528$.

[6] B. Brighi, On a similarity boundary layer equation, Z. Anal. Anwendungen 21 (2002), no. 4, 931-948.

[7] B. Brighi, A. Fruchard and T. Sari, On the Blasius problem, preprint (http://hal.archives-ouvertes.fr/hal-00114834).

[8] B. Brighi and J.-D. Hoernel, On similarity solutions for boundary layer flows with prescribed heat flux, Math. Methods Appl. Sci. 28 (2005), no. 4, 479-503.

[9] B. Brighi and J.-D. Hoernel, Recent advances on similarity solutions arising during free convection, in Progress in Nonlinear Differential Equations and Their Applications 63 Birkhäuser Verlag Basel/Switzerland 2005, 83-92.

[10] B. Brighi and J.-D. Hoernel, Asymptotic behavior of the unbounded solutions of some boundary layer equation, Arch. Math. (Basel) 85 (2005), no. 2, 161-166.

[11] B. Brighi and J.-D. Hoernel, On a general similarity boundary layer equation, to appear in Acta Math. Univ. Comenian. (http://arxiv.org/abs/math/0601385 ; http://hal.archives-ouvertes.fr/hal-00017451).

[12] B. Brighi and T. Sari, Blowing-up coordinates for asimilarity boundary layer equation, Discrete Contin. Dyn. Syst. (Serie A) 12 (2005), no. 5, 929-948.

[13] M. A. Chaudhary, J.H. Merkin and I. Pop, Similarity solutions in free convection boundary-layer flows adjacent to vertical permeable surfaces in porous media: I prescribed surface temperature, European J. Mech. B Fluids 14 (1995), no. 2, 217237.

[14] M. A. Chaudhary, J.H. Merkin and I. Pop, Similarity solutions in free convection boundary-layer flows adjacent to vertical permeable surfaces in porous media: II prescribed surface heat flux, Heat and Mass Transfer 30 (1995), 341-347. 
[15] P. Cheng and W. J. Minkowycz, Free-convection about a vertical flat plate embedded in a porous medium with application to heat transfer from a dike, J. Geophys. Res. 82 (1977), no. 14, 2040-2044.

[16] E. I. Ene and D. Poliševski, Thermal flow in porous media, D. Reidel Publishing Company, Dordrecht, 1987.

[17] M. Guedda, Similarity solutions of differential equations for boundary layer approximations in porous media, J. Appl. Math. Phys. (ZAMP) 56 (2005), 749-762.

[18] J.-S. Guo and J.-C. Tsai, The structure of the solutions for a third order differential equation in boundary layer theory, Japan J. Indust. Appl. Math. 22 (2005), no. 3, $311-351$.

[19] J. H. Merkin, G. Zhang, On the similarity solutions for free convection in a saturated porous medium adjacent to impermeable horizontal surfaces, Wärme und Stoffübertr. 25 (1990), pp.179-184.

[20] J.-C. Tsai, Similarity solutions for boundary layer flows with prescribed surface temperature, Appl. Math. Lett., 21, 1 (2008) 67-73.

[21] J.-C. Tsai and C.-A. Wang, A note on similarity solutions for boundary layer flows with prescribed heat flux, Math. Methods Appl. Sci. 30, 12 (2007), 1453-1466.

[22] R. A. Wooding, Convection in a saturated porous medium at large Rayleigh number or Peclet number, J. Fluid. Mech. 15 (1963), 527-544.

Bernard BRIGHI

Laboratoire de Mathématiques, Informatique et Applications

Université de Haute Alsace

4, rue des Frères Lumière

68093 Mulhouse CEDEX France

Bernard.Brighi@uha.fr

Senoussi GUESMIA

Service de Métrologie Nucléaire

Universite Libre de Bruxelles (C.P. 165)

50, Av. F.D. Roosevelt, B-1050 Brussels, Belgium.

sguesmia@ulb.ac.be

Laboratoire de Mathématiques, Informatique et Applications

Université de Haute Alsace

4, rue des Frères Lumière

68093 Mulhouse CEDEX France

Senoussi.Guesmia@uha.fr 